\title{
QUALIDADE DE VIDA DOS PROFISSIONAIS ATUANTES NO CENTRO CIRÚRGICO
}

DOI: $10.22289 / 2446-922 X . V 3 N 2 A 12$

\author{
Luciana Maria Pereira Soares \\ Victor Constante Oliveira ${ }^{1}$ \\ Luíza Araújo Amâncio Sousa
}

\section{RESUMO}

Este estudo descritivo exploratório teve por objetivo verificar quais os fatores estressantes que interferem na qualidade de vida dos profissionais que atuam no Centro Cirúrgico de diferentes hospitais. Os dados foram coletados entre os meses de fevereiro e março de 2017, por meio de um questionário contendo uma questão norteadora com 22 profissionais, sendo 13 técnicos de enfermagem, cinco enfermeiros e quatro médicos. Aplicou-se análise de conteúdo temática resultando em seis categorias. Os resultados apontam que a maioria era do sexo feminino, casados, com idade média entre 26 e 45 anos, e técnicos de enfermagem. $\mathrm{Na}$ avaliação geral da qualidade de vida, os dados obtidos revelaram que o fator que mais gera estresse na vida desses profissionais é o tipo de ambiente fechado, sendo que os demais fatores variaram de acordo com a categoria profissional. Conclui-se que os profissionais de enfermagem vivenciaram o estresse relacionado a diversos fatores de natureza objetiva.

Palavras-chave: Saúde ocupacional; Condições de trabalho; Fatores estressores; Centro cirúrgico hospitalar.

\section{ABSTRACT}

This exploratory descriptive study aimed to verify which stress factors interfere in the quality of life of the professionals who work in the Surgical Center of different hospitals. The data were collected between February and March 2017, through a questionnaire containing a guiding question with 22 professionals, 13 nursing technicians, five nurses and four doctors. Thematic content analysis was applied resulting in six categories. The results indicate that the majority were female, married, with an average age between 26 and 45 years, and nursing technicians. In general evaluation of the quality of life, the data obtained revealed that the factor that causes the most stress in the life of these professionals is the type of closed environment, and the other factors varied according to the professional category. It was concluded that the nursing professionals experienced the stress related to several objective factors.

Keywords: Occupational health; Work conditions; Stressors; Hospital surgical center.

\footnotetext{
${ }^{1}$ Endereço eletrônico de contato: victor.biologia@hotmail.com
}

Recebido em 20/09/2017. Aprovado pelo Conselho Editorial e aceito para publicação em 19/10/2017.

Rev. Psicol Saúde e Debate. Dez., 2017:3(2):159-170. 


\section{INTRODUÇÃO}

O centro cirúrgico (CC) é um setor hospitalar que conta com uma equipe multiprofissional atuante como: médicos anestesiologistas, médicos cirurgiões, instrumentadores cirúrgicos, enfermeiro coordenador e técnicos de enfermagem; equipamentos e instrumentos apropriados para a realização do processo cirúrgico, oferecendo condições que proporcionam qualidade e segurança para os profissionais de enfermagem e para que atendam as necessidades dos pacientes antes, durante e após a cirurgia (Rodrigues \& Souza, 1993; Martins, 2013).

O CC é caracterizado como uma área física fechada, de risco e com vários regulamentos e rotinas (Stumm, Maçalai \& Kirchner, 2006). Os procedimentos de trabalho dependem da interação entre os profissionais que atuam na referida unidade, devendo acontecer de forma harmoniosa (Caregnato, 2002), além de apresentar conhecimento científico sobre os procedimentos cirúrgicos, responsabilidade, habilidade técnica, e estabilidade emocional (Stumm et al., 2006), pois estes profissionais têm tolerado jornadas de trabalho cada vez mais prolongadas dentro desse setor, com proporção inadequada de pacientes por profissionais qualificados, turnos alternados, baixa remuneração, exposição a substâncias tóxicas e fatores de risco relacionados ao ambiente, levando a uma circunstância conhecida como sobrecarga de trabalho (Oler, Jesus, Barboza, \& Domingos, 2005).

Segundo Ferrareze, Ferreira e Carvalho (2006), o estresse ocupacional, ao qual trabalhador moderno está exposto, tem se destacado como assunto de suma importância, principalmente em algumas profissões como, por exemplo, os profissionais que atuam no CC. O estresse ocupacional é um estado em que ocorre desgaste anormal do organismo humano e/ou diminuição da capacidade de trabalho, devido basicamente à incapacidade prolongada de o indivíduo tolerar, superar ou se adaptar às exigências de natureza física e emocional existentes em seu ambiente de trabalho ou de vida (Schmidt, Dantas, Marziale, \& Laus, 2009).

Desta forma, a exposição aos riscos físicos, químicos, biológicos, ergonômicos e a sobrecarga de trabalho aumenta cada vez mais o nível de estresse dos profissionais, pois o CC exige um alto grau de responsabilidade, além de o profissional permanecer nas salas de cirurgia por um longo tempo, acompanhando e atuando nos diversos procedimentos cirúrgicos, de diferentes doenças e com grau de complexidade variado, desde os mais simples aos mais graves. Tais condições resultam no (des)equilíbrio físico e emocional, insatisfação, com consequente queda da produtividade, e acidentes de trabalho (Miranda, 2006). Diante disso, o objetivo do presente estudo foi verificar quais os fatores estressantes que interferem na qualidade de vida dos profissionais que atuam no Centro Cirúrgico. 


\section{MÉTODOS}

Trata-se de um estudo exploratório, descritivo e transversal sobre a avaliação dos fatores que interferem na qualidade de vida da equipe de profissionais atuantes no centro cirúrgico. O estudo de natureza descritiva-exploratório permite ao pesquisador detalhar e analisar um determinado problema propiciando a esse maior conhecimento em torno do tema, além de definir objetivos e buscar maiores informações sobre determinado assunto estudado (Cervo \& Bervian, 1996).

O presente estudo foi desenvolvido em Centros Cirúrgicos de diferentes hospitais da Região do Alto Paranaíba, Estado de Minas Gerais, Brasil, por compreender de um setor que possui características próprias de trabalho. Os encontros com os profissionais aconteceram após as sessões de cirurgia, mantendo a privacidade e conforto para os sujeitos. Fizeram parte do estudo vinte e dois sujeitos ( $n=22$ ), incluindo técnicos de enfermagem, enfermeiros e médicos, os quais foram selecionados de forma aleatória, no período de fevereiro e março de 2017, por atenderem aos seguintes critérios de seleção: trabalhar no centro cirúrgico há mais de dois anos, ter jornada de trabalho igual ou superior a 44 horas semanais, ter idade maior que 18 anos, manifestar interesse em falar sobre o tema proposto, aceitar formalmente participar da pesquisa e assinar o TCLE (Termo de consentimento Livre Esclarecido).

Os dados foram obtidos por meio de entrevistas com uma questão norteadora: "Para você, quais são os fatores que geram estresse durante o seu trabalho no centro cirúrgico?". Esta pergunta por sua vez, visou a obter dados quanto à percepção da equipe quanto à exposição dos fatores estressantes.

Os dados foram analisados a partir da leitura da questão norteadora, e seguiram os seguintes passos: codificação dos dados com a identificação dos trechos que abordavam a questão pesquisada; formação das categorias temáticas; elaboração da identificação do conjunto de expressões que representam os fatores estressantes aos quais a equipe do centro cirúrgico está exposta durante sua jornada de trabalho. O projeto da pesquisa foi apreciado pelo Comitê de Ética da Faculdade Patos de Minas - FPM e aprovado pelo parecer oㅜ 2.071.876. 


\section{RESULTADOS}

Os dados coletados foram categorizados segundo objetivo da pesquisa, buscandose agrupar as respostas semelhantes. Os resultados foram analisados quantiqualitativamente e apresentados em forma de figuras, tabelas e discursiva. Para compor a amostra do estudo foram entrevistados vinte e dois sujeitos $(n=22)$ atuantes no centro cirúrgico (CC) de diferentes hospitais com aqueles possuíam os seguintes vínculos empregatícios: Técnicos de enfermagem, enfermeiros e médicos (Figura 1). Durante o período estabelecido para coleta de dados foram excluídos os trabalhadores de férias, licença médica e licença gestante. Conforme pode ser observado na Figura 1, os participantes do estudo eram predominantemente Técnicos de enfermagem (59\%), sendo que as demais proporções de enfermeiros $(22,8 \%)$ e médicos $(18,2 \%)$ foram semelhantes.

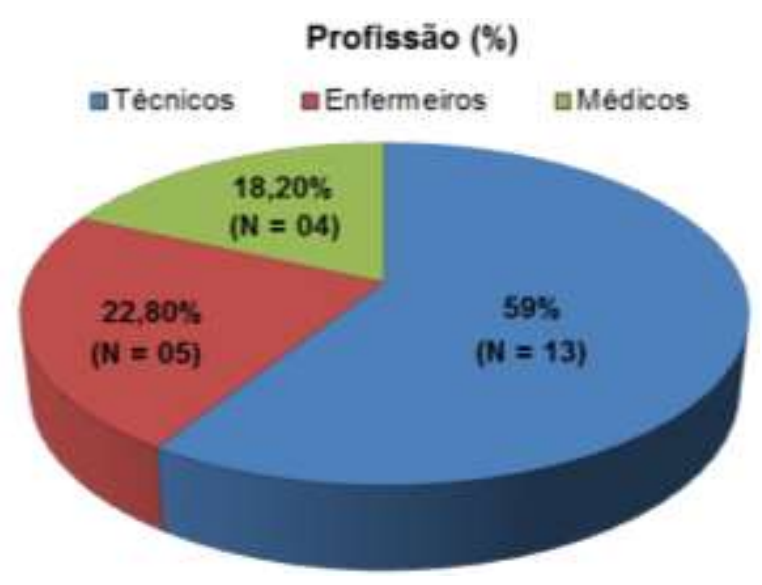

Figura 1: Distribuição total dos profissionais entrevistados que atuam no Centro Cirúrgico em diferentes hospitais da Região do Alto Paranaíba, Estado de Minas Gerais.

Os resultados foram analisados em função da amostra estudada $(n=22)$, agrupados de forma a atender os objetivos deste estudo. A descrição dos sujeitos entrevistados quanto ao sexo, faixa etária, estado civil, grau de instrução e remuneração é apresentada na Tabela 1 .

Rev. Psicol Saúde e Debate. Dez., 2017:3(2):159-170. 
Descrição das variáveis sociodemográficas da amostra avaliada.

\begin{tabular}{lll}
\hline & Variável & N (\%) \\
\hline Sexo & & \\
& Masculino & $06(27,2)$ \\
& Feminino & $16(72,8)$
\end{tabular}

\section{Faixa Etária}

$\begin{array}{cl}18 \text { a } 25 \text { anos } & 02(9,0) \\ 26 \text { a } 35 \text { anos } & 08(36,4) \\ 36 \text { a } 45 \text { anos } & 08(36,4) \\ \text { Acima de } 45 \text { anos } & 04(18,2)\end{array}$

\section{Estado Civil}

$\begin{array}{cl}\text { Casado } & 17(77,3) \\ \text { Solteiro } & 03(13,7) \\ \text { Separado } & 02(9,0) \\ \text { Viúvo } & 00(0,0) \\ \text { Outros } & 00(0,0)\end{array}$

Formação

$2^{\circ}$ Grau completo $08(36,4)$

Superior incompleto $06(27,2)$

Superior completo $\quad 08(36,4)$

\section{Faixa Salarial}

$\begin{array}{cl}R \$ 1200,00 \text { a } R \$ 1999,00 & 07(31,8) \\ R \$ 2000,00 \text { a } R \$ 2999,00 & 06(27,2) \\ R \$ 3000,00 \text { a } R \$ 3999,00 & 04(18,2) \\ R \$ 4000,00 \text { a } R \$ 4999,00 & 01(4,6) \\ \text { Acima de } R \$ 5000,00 & 04(18,2)\end{array}$

Conforme apresentado na Tabela 1, os participantes do estudo eram predominantemente do sexo feminino (72,8\%), com idade compreendida entre 26 e 45 anos (72,8\%). Além disso, a média geral de faixa etária foi de 36 anos. Quanto ao estado civil dos profissionais, $17(77,3 \%)$ eram casados, três (13,7\%) solteiros, dois $(9,0 \%)$ estavam separados dos cônjuges e não teve viúvos. Em relação à escolaridade dos profissionais, oito Rev. Psicol Saúde e Debate. Dez., 2017:3(2):159-170. 
$(36,4 \%)$ possuíam o $2^{\circ}$ grau completo, seis $(27,2 \%)$ superior incompleto, e oito $(36,4 \%)$ superior completo, sendo que a maioria tem remuneração entre $R \$ 1200,00$ e $R \$ 2999,00$.

Como descrito na metodologia, a qualidade de vida dos profissionais que atuam no $\mathrm{CC}$, e os fatores que geram estresse neste ambiente de trabalho foram avaliados por meio da questão norteadora presente nos questionários. A análise das falas levou a construção de seis categorias temáticas que mais geram estresse no cotidiano destes profissionais: Relações interpessoais com a equipe multiprofissional, e com os familiares dos pacientes; falta de materiais e equipamentos, incluindo os estragos que impossibilitam a utilização; tipo de ambiente fechado, no qual estão sujeitos a diversos procedimentos cirúrgicos de baixa, média e alta complexidade, incluindo as situações de emergência; falta de assiduidade e pontualidade, tanto dos profissionais como dos pacientes; baixa remuneração e desvalorização; e sobrecarga de trabalho.

Vale ressaltar que, por se tratar de uma pergunta discursiva, cada sujeito entrevistado pode escrever várias respostas, inclusive mais de uma resposta relacionada a uma mesma categoria temática. Assim, as respostas que se enquadraram na mesma categoria, foram reunidas. A Tabela 2 Apresenta a proporção dos fatores geradores de estresse no CC distribuídos nas seis categorias e separados por profissão: Técnicos, enfermeiros e médicos.

\section{TABELA 2}

Descrição das variáveis geradoras de estresse nos profissionais atuantes no Centro Cirúrgico ( $\mathrm{n}=22)$, separados por profissão.

\begin{tabular}{lccc}
\hline \multicolumn{1}{c}{ Fator } & $\begin{array}{c}\text { Técnicos } \\
\mathbf{n ~ ( \% )}\end{array}$ & $\begin{array}{c}\text { Enfermeiros } \\
\mathbf{n ~ ( \% )}\end{array}$ & $\begin{array}{c}\text { Médicos } \\
\mathbf{n}(\%)\end{array}$ \\
\hline Relações interpessoais & $8(19,0)$ & $6(24,0)$ & $2(13,3)$ \\
Falta de Materiais e Equipamentos & $4(9,5)$ & $4(16,0)$ & $3(20,0)$ \\
Tipo de ambiente fechado & $13(31,0)$ & $6(24,0)$ & $6(40,0)$ \\
Falta de assiduidade e pontualidade & $5(11,9)$ & $4(16,0)$ & $3(20,0)$ \\
Baixa remuneração e desvalorização & $0(0,0)$ & $1(4,0)$ & $0(0,0)$ \\
Sobrecarga de trabalho & $12(28,6)$ & $4(16,0)$ & $1(6,7)$ \\
Total $^{\text {a }}$ & $\mathbf{4 2 ( 1 0 0 )}$ & $\mathbf{2 5 ( 1 0 0 )}$ & $\mathbf{1 5 ( 1 0 0 )}$ \\
\hline
\end{tabular}

$\mathrm{n}=$ Número de respostas por grupo de fatores geradores de estresse.

a Considerando mais de uma resposta por sujeito entrevistado. 
Como pode ser observado na Tabela 2, o tipo de ambiente fechado foi o fator gerador de estresse mais citado por ambos profissionais: Técnicos (31\%), Enfermeiros (24\%) e médicos (40\%). Os demais fatores variaram de acordo com a categoria profissional. Com relação aos técnicos de enfermagem, o segundo fator que mais gera estresse é a sobrecarga de trabalho $(28,6 \%)$, seguido das relações interpessoais $(19 \%)$, falta de assiduidade e pontualidade $(11,9 \%)$, e falta de materiais e equipamentos $(9,5 \%)$, não tendo nenhuma citação referente à baixa remuneração e desvalorização. Em relação aos enfermeiros, as relações interpessoais apresentaram a mesma proporção do tipo de ambiente $(24 \%)$, seguido da falta de materiais e equipamentos, falta de assiduidade e pontualidade e sobrecarga de trabalho (16\%), e baixa remuneração e desvalorização (4\%). Quanto aos médicos, os outros fatores que mais causam estresse é a falta de materiais e equipamentos e falta de assiduidade e pontualidade (20\%), seguido das relações interpessoais $(13,3 \%)$ e sobrecarga de trabalho $(6,7 \%)$, não tendo nenhuma citação referente à baixa remuneração e desvalorização.

A comparação entre os seis grupos de fatores geradores de estresse e as categorias de profissionais pode melhor ser observada na Figura 2.

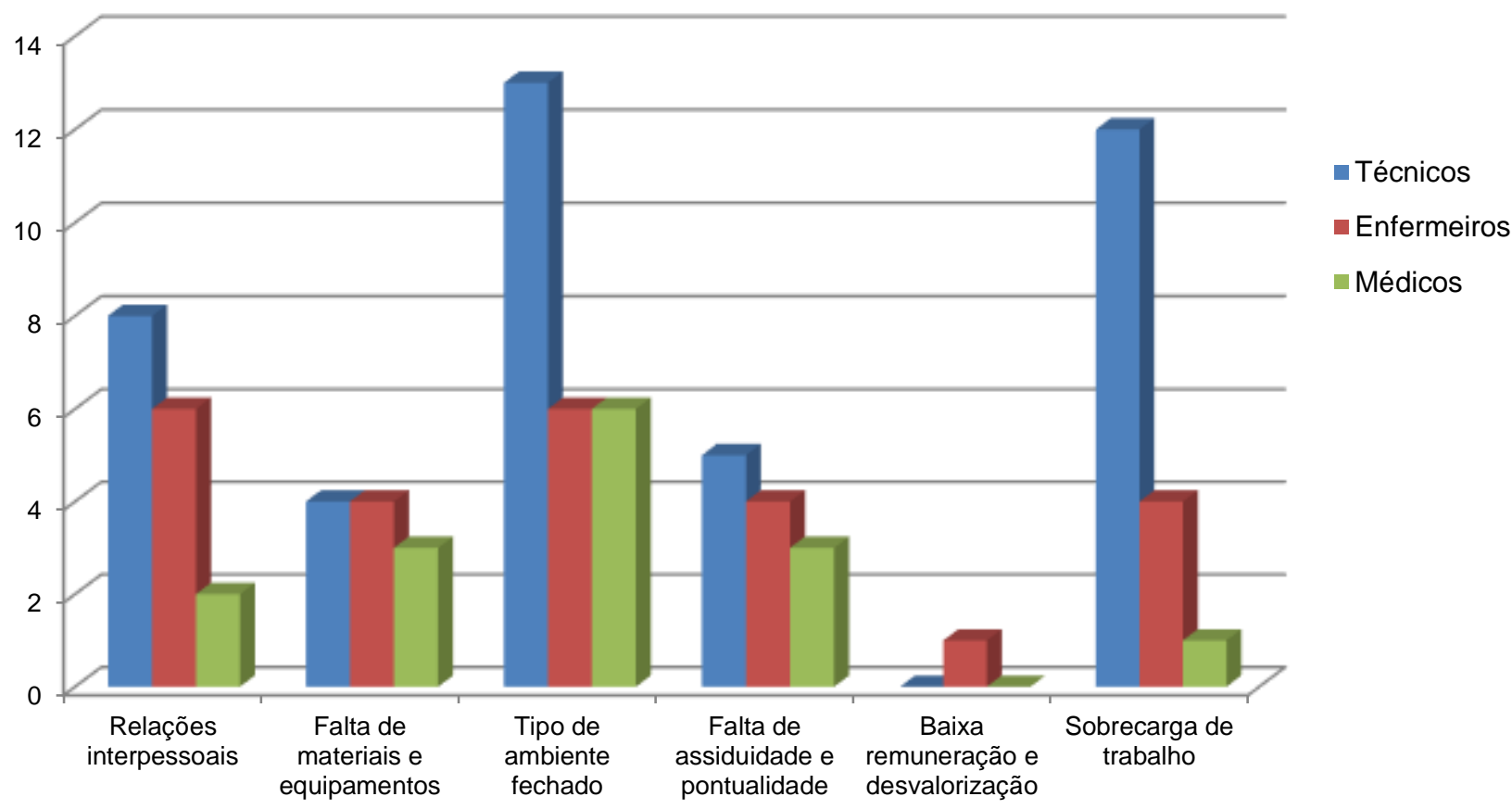

Figura 2: Representação dos fatores geradores de estresse dos profissionais entrevistados que atuam no Centro Cirúrgico em diferentes hospitais da Região do Alto Paranaíba, Estado de Minas Gerais. 


\section{DISCUSSÃo}

Alguns estudos sobre a qualidade de vida têm sido realizados com diferentes grupos e em diferentes aspectos (Totoro, Sousa, Martins, Chacur, \& Souza 2017; Viana \& Antoniassi Junior, 2017), reforçando a importância de verificar quais os fatores que interferem. Nos resultados obtidos neste estudo, em relação ao perfil da qualidade de vida dos profissionais que atuam no Centro Cirúrgico (CC) em diferentes hospitais da Região do Alto Paranaíba, Estado de Minas Gerais, confirmam os achados de outras pesquisas, onde o profissional mais envolvido pertence ao sexo feminino e atua como técnico de enfermagem (Costa, Morita \& Martinez, 2000; Pereira \& Fávero, 2001). Essas informações reafirmam que tradicionalmente, as funções relacionadas ao cuidado dos pacientes, com suas especialidades tecnológicas próprias de auxiliar, higienizar, alimentar, fornecerem os elementos indispensáveis ao bom desenvolvimento do doente, seguindo os padrões da divisão social do trabalho, sempre esteve confiado ás mulheres (Oler et al., 2005).

Em relação à faixa etária dos profissionais entrevistados, a média de idade foi de 36 anos. Já para a variável estado civil os resultados obtidos apontam que a maioria dos profissionais entrevistados eram casados (77,3\%). É importante destacar que o grupo entrevistado, constitui-se principalmente de profissionais de faixa etária relativamente mais baixa, sendo a maioria casados, o que nos leva a crer que a socialização, isto é, fazer amigos e o relacionamento com a família, reflete na satisfação deste grupo com a dimensão estudada.

Com relação à formação, a proporção de profissionais com $2^{\circ}$ grau completo, superior incompleto e superior completo foi semelhante, $36,4 \%, 27,2 \%$ e $36,4 \%$ respectivamente. No entanto, observou-se que maioria dos profissionais entrevistados recebe remuneração entre $R \$ 1200,00$ e $R \$ 1999,00$. Esses dados eram esperados, visto que a maior parte dos entrevistados exerce a função de técnicos de enfermagem. No entanto, no presente estudo, apenas um entrevistado mencionou a baixa remuneração e desvalorização como fator causador de estresse. Assim, embora a baixa remuneração e desvalorização possa causar desmotivação nesses profissionais, acredita-se que os outros fatores sejam mais importantes no sentido que causar estresse dentro do CC.

Após analisar os dados sociodemográficos desses profissionais, avaliamos os fatores que mais causam estresse durante sua atuação no CC, e observamos que o fator que mais causa estresse em ambas as categorias profissionais entrevistadas é o tipo de ambiente fechado, enquanto houve variação dos demais fatores de acordo com a categoria profissional. É conhecido que o CC é um setor fechado e com particularidades próprias, onde é necessário limitar o contato pessoal de fora desse setor, não ocorrendo, portanto a relação interpessoal 
com os outros setores do hospital. O contato com o ambiente externo geralmente ocorre por via telefônica e por meio do recebimento de materiais e recepção de paciente (Silva \& Popov, 2010).

Segundo Passos, Silva e Carvalho (2010), o fato dos profissionais que atuam CC exercerem as suas atividades funcionais em ambientes fechados, o estresse pode ser um fator presente, considerando que este setor induz os trabalhadores a se sentirem excluídos de acontecimentos e atividades que ocorram no ambiente hospitalar, como a troca de experiências entre os profissionais e pouca participação em treinamentos levando, muitas vezes, à defasagem de atualizações sobre a prática nas suas atividades diárias.

Em relação aos técnicos de enfermagem, os outros fatores que mais causam estresse no CC é a sobrecarga de trabalho, relações interpessoais, falta de assiduidade e pontualidade e falta de materiais e equipamentos respectivamente. Assim, com base nestes resultados, é possível observar que além do fato de trabalharem em um ambiente fechado como o CC, a sobrecarga de trabalho e as relações interpessoais é o que mais induz estes profissionais ao estresse.

Quanto aos enfermeiros, outro fator que mais causa estresse no CC são as relações interpessoais, seguidos pela falta de materiais e equipamentos, falta de assiduidade e pontualidade e sobrecarga de trabalho na mesma proporção, e a baixa remuneração e desvalorização. Diante destes resultados é possível observar que os fatores que mais causam estresse nestes profissionais é trabalhar num ambiente fechado, e as relações interpessoais. Contudo, os outros fatores que mais causam estresse nos médicos dentro do CC é a falta de materiais e equipamentos e a falta de assiduidade e pontualidade na mesma proporção, seguido das relações interpessoais e sobrecarga de trabalho.

Meirelles e Zeitoune (2003) afirmam que algumas características da organização do trabalho hospitalar induzem os profissionais do CC ao estresse, entre elas, as jornadas de trabalho extensas e os ritmos acelerados de trabalho, a conduta repressora e autoritária de uma hierarquia rígida e vertical, a fragmentação das tarefas, os riscos ocupacionais e, consequentemente, de quem o realiza. Estudos relatam que a falta de descanso e de diversão, na maioria das vezes, leva o indivíduo ao estresse que é considerado um problema negativo, de caráter perceptivo, resultante da incapacidade de suportar as pressões no ambiente de trabalho (Lentz, Costenaro, Gonçalves, \& Nassar, 2000).

A falta de planejamento correto quanto aos procedimentos realizados no CC também pode desencadear conflitos entre a equipe multiprofissional, gerando estresse e impossibilitando a assistência adequada (Silva \& Popov, 2010). Além disso, a falta de assiduidade e pontualidade de alguns profissionais no $\mathrm{CC}$ exigem que realizem inúmeras 
tarefas que deveriam ser repartidas com outros membros da equipe. Isso resulta no aumento das exigências físicas e emocionais, gerando estresse físico e/ou mental e influenciando na qualidade destes profissionais (Rodrigues, 2012).

Com relação às relações interpessoais, Monteiro, Oliveira, Ribeiro, Grisa, e Agostini (2013) relatam como origem do estresse e muito desgastante o relacionamento entre a equipe multiprofissional e familiares dos pacientes no CC. Muitas das vezes, os familiares dos pacientes muitas vezes atribuem os erros cirúrgicos aos profissionais responsáveis, pois são eles que passam com o paciente a maior parte do tempo e são os responsáveis pelos cuidados. Neste contexto, é fundamental ter compreensão e empatia, além de respeitar as diferenças entre os membros da equipe. Quanto aos familiares, responder na medida do possível os questionamentos, minimizando a angústia e sofrimento, pois é o profissional que mantém maior contato e por mais tempo com paciente.

Outros autores afirmaram que os equipamentos e materiais que não funcionam ou que funcionam inadequadamente durante os procedimentos cirúrgicos, utilização de materiais impróprios ou estragados, ausência de material e equipamentos em quantidade insuficiente para os pacientes, uso de produtos inadequados e improvisos, são mencionados como estressores para o grupo cirúrgico, sobrecarregando a atuação do ajudante e causando estresse. Estas situações estressantes interferem significativamente no equilíbrio emocional do profissional (Caregnato \& Lautert, 2005; Silva \& Popov, 2010).

Com base nos resultados expostos no presente estudo, seria importante considerar as necessidades individuais destes profissionais, bem como sua aptidão de superação, adaptação e reação nas diferentes situações da vida pessoal, social e profissional, uma vez que cada indivíduo características próprias de lidar com situações conflitantes que desencadeiam o estresse. Inicialmente, os fatores de estresse identificados neste estudo servem de apoio para gerar reflexões acerca da qualidade de vida dos profissionais relacionadas às condições do ambiente de trabalho, implementando programas de atenção à saúde do trabalhador, organização racional e valorização do trabalho, bem como a interação multiprofissional.

\section{CONSIDERAÇÕES FINAIS}

Diante dos resultados encontrados no presente estudo, conclui-se que os profissionais atuantes no centro cirúrgico na Região do Alto Paranaíba, Estado de Minas Gerais vivenciaram o estresse relacionado a fatores de natureza objetiva como: as relações interpessoais, falta de materiais e equipamentos, tipo de ambiente 
fechado, falta de assiduidade e pontualidade, sobrecarga de trabalho, e em menor proporção, a baixa remuneração e desvalorização.

Embora os objetivos propostos neste estudo foram alcançados, é indispensável considerar os resultados em sua singularidade, uma vez que representa a realidade de uma pequena parte de profissionais atuantes no CC de diferentes. Novos estudos devem ser realizados a fim de identificar os principais fatores que geram estresse nos profissionais que atuam no CC em hospitais de diferentes regiões do país, para que busque alternativas que minimizem ou até mesmo elucidem os fatores que interferem na sua qualidade de vida.

\section{REFERÊNCIAS}

Caregnato, R. C. A. (2002). Estresse da equipe multiprofissional na sala de cirurgia: um estudo de caso. Tese de Doutorado, Universidade Federal do Rio Grande do Sul, Porto Alegre, RS, Brasil.

Caregnato, R. C. A., \& Lautert, L. O. (2005) estresse da equipe multiprofissional na Sala de Cirurgia. Revista Brasileira de Enfermagem, 58 (5), 545-50.

Cervo, A.L, \& Bervian, P.A. (1996). A pesquisa: noções gerais. São Paulo: Makron Books.

Costa, E. S., Morita, I. \& Martinez, M. A. (2000). Percepção dos efeitos do trabalho em turnos sobre a saúde e a vida social em funcionários da enfermagem em um hospital universitário do Estado de São Paulo. Cadernos de saúde pública, 16 (02), 553-555.

Ferrareze, M. V. G., Ferreira, V., \& Carvalho, A. M. P. (2006). Percepção do estresse entre enfermeiros que atuam em Terapia Intensiva. Acta Paulista de Enfermagem, 19 (3), 310-5.

Lentz, R. A., Costenaro, R. G. S., Gonçalves, L. H., \& Nassar, S. M. (2000). O profissional de enfermagem e a qualidade de vida: uma abordagem fundamentada nas dimensões propostas por Flanagan. Revista Latino-Americana de Enfermagem, 8 (4), 7-14.

Martins, F. Z (2013). Atividades gerenciais do enfermeiro em centro cirúrgico. Dissertação de mestrado, Universidade Federal do Rio Grande do Sul, Porto Alegre, RS, Brasil.

Meirelles, N. F., \& Zeitoune, R. C. G. (2003). Satisfação no trabalho e fatores de estresse da equipe de enfermagem de um centro cirúrgico oncológico. Escola Anna Nery Revista de Enfermagem, 7(1), 78-88.

Miranda, E. P. (2006). Qualidade de vida de profissionais de enfermagem que atuam em centro cirúrgico, Dissertação de mestrado, Universidade Federal da Paraíba, João Pessoa, PB, Brasil. 
Monteiro, J. K., Oliveira, A. L. L., Ribeiro, C. S, \& Grisa, G. H. (2013). Agostini, N. Adoecimento psíquico de trabalhadores de unidades de terapia intensiva. Psicologia Ciência e Profissão, 33 (2), 366-79.

Oler, F. G., Jesus, A. F. D., Barboza, D. B., \& Domingos, N. A. M. (2005). Qualidade de vida da equipe de enfermagem do centro cirúrgico. Arquivos de Ciências da Saúde, 12 (2), 10210.

Passos, J. B., Silva, E. L., \& Carvalho, M. M. C. (2010). Estresse no centro cirúrgico: uma realidade dos profissionais de enfermagem. Revista de Pesquisa em Saúde, 11(2), 35-8.

Pereira, M. C. A. \& Fávero, N. A motivação no trabalho da equipe de enfermagem. Revista Latino-Americana de Enfermagem, 9 (4), 7-12.

Rodrigues, R. A. P. \& Sousa, F. A. E. F (1993). O trabalho da enfermagem em centro cirúrgico: análise de depoimentos. Revista Latino-Americana de Enfermagem, 1 (2), 21-34.

Rodrigues, T. D. F. Fatores estressores para a equipe de enfermagem da unidade de terapia intensiva. Revista Mineira de Enfermagem, 16 (3), 454-462.

Schmidt, D. R. C., Dantas, R. A. S., Marziale, M. H. P., \& Laus, A. M. (2009). Estresse ocupacional entre profissionais de enfermagem do bloco cirúrgico. Texto \& ContextoEnfermagem, 18 (2), 330-337.

Silva, P. P., \& Popov, D. C. S. (2010). Estresse da equipe de enfermagem no centro cirúrgico. Revista de Enfermagem UNISA, 11 (2),125- 30.

Stumm, E. M. F., Maçalai, R. T., \& Kirchner, R. M. (2006). Dificuldades enfrentadas por enfermeiros em um centro cirúrgico. Texto \& Contexto Enfermagem, 15 (3), 464-71.

Totoro, R., Sousa, M., Martins, R., Chacur, E., \& Souza, F. (2017). Avaliação da qualidade de vida dos usuários de um núcleo de apoio à saúde da família-NASF. Psicologia e Saúde em debate, 3(1), 50-67.

Viana, A., \& Junior, G. A. (2017). Qualidade de vida em idosos praticantes de atividades físicas. Psicologia e Saúde em debate, 3(1), 87-98. 